\title{
Reduced basis numerical homogenization method for the multiscale wave equation
}

\author{
Assyr Abdulle, Yun Bai, and Timothée Pouchon \\ ANMC, Section de Mathématiques, École Polytechnique Fédérale de Lausanne, \\ Switzerland, \{assyr.abdulle, yun. bai, timothee. pouchon $\}$ @epfl. ch*
}

\begin{abstract}
A reduced basis numerical homogenization method for the wave equation in heterogeneous media is presented. The method is based on a macroscopic discretization of the physical domain with input data recovered from microscopic problems solved by using reduced basis techniques. A priori error analysis is discussed and the convergence rates are verified by numerical experiments that also illustrate the performance of the method.
\end{abstract}

\section{Introduction}

Consider the wave equation in a polygonal domain $\Omega \subset \mathbb{R}^{d}$

$$
\partial_{t}^{2} u^{\varepsilon}(t, x)-\nabla \cdot\left(a^{\varepsilon}(x) \nabla u^{\varepsilon}(t, x)\right)=f(t, x) \text { in }(0, T) \times \Omega,
$$

where the tensor $a^{\varepsilon}$ has rapid oscillations on a small scale $\varepsilon \ll \operatorname{diam}(\Omega)$. This equation enters the modeling of various applications such as the wave propagation in composite material, seismic imaging or medical ultrasound imaging. Together with (1.1), we set zero Dirichlet boundary condition and initial conditions $u^{\varepsilon}(0, \cdot)=g_{0}, \partial_{t} u^{\varepsilon}(0, \cdot)=g_{1}$. Furthermore, assume that $a^{\varepsilon}$ is in $\left(L^{\infty}(\Omega)\right)^{d \times d}$, symmetric, uniformly elliptic and bounded. It follows from [15] that the problem (1.1) is well-posed, provided sufficient regularity of the functions $f, g_{0}$ and $g_{1}$.

It is well-known that standard numerical methods based on scale resolution for (1.1) are prohibitively expensive if $\varepsilon$ is small. Fortunately, there is a well-developed mathematical theory, the homogenization theory, that describes an effective equation that captures the macroscopic behavior $[7,14]$. In particular for the wave equation, the notions of $G$ or $H$-convergence [16] have been used in [9] to show that $\left\{u^{\varepsilon}\right\}$ converges in a weak sense as $\varepsilon \rightarrow 0$ to the homogenized solution $u^{0}$ which satisfies the homogenized equation

$$
\partial_{t}^{2} u^{0}(t, x)-\nabla \cdot\left(a^{0}(x) \nabla u^{0}(t, x)\right)=f(t, x) \quad \text { in } \quad(0, T) \times \Omega .
$$

We note that $a^{0}(x)$ is obtained via micro problems that are usually not explicitly known and various numerical strategies have been proposed in the

\footnotetext{
*This work is partially supported by the Swiss National Foundation Grant 200021_134716
} 
past several years to compute an approximation of $u^{\varepsilon}$ or $u^{0}$ (we refer to $[13,1,5]$ for a literature review on such numerical strategies). Here we focus on the finite element heterogeneous multiscale method (FE-HMM) introduced in $[11,2,12]$ and first proposed for the wave equation in [1].

The basic FE-HMM can be summarized as follows: at the micro scale an effective solver based on numerical quadrature is defined. This solver relies on effective data (located at quadrature points) that needs to be recovered by micro cell problems, based on the heterogeneous tensor $a^{\varepsilon}$. A complete numerical analysis of this method including the contribution of micro errors, modeling error and macro error has been given in [1]. We note that the complexity of this method is independent of $\varepsilon$ as the macro partition is independent of this small scale. However the issue for the FE-HMM is the large amount of repeated micro computations that need to be performed. This is a consequence of the fact that these micro problems are located around macroscopic quadrature points. Thus for optimal convergence, as shown in the a priori error analysis in $[2,1]$, both the number of the micro problems and their complexity increase simultaneously as the macro mesh is refined.

The aforementioned issue triggered the development of a reduced order modeling for the FE-HMM $[3,4]$. The main idea for these new methods, called reduced basis heterogeneous multiscale method (RB-FE-HMM), is to combine numerical homogenization method with reduced basis techniques for the micro-problems. The reduced basis method (see [17] and the references therein) gives a methodology to construct low dimensional subspaces of the solutions of parametrized partial differential equations. This can be exploited in numerical homogenization in an offline stage to construct a low dimensional approximation for the micro problems [8,3]. The approximation of the effective data in the macro RB-FE-HMM (the offline stage) is then computed in this low dimensional subspace at a cost comparable to a standard FEM with numerical quadrature for single-scale problems. In this paper, we discuss the use of reduced basis for the numerical homogenization of the wave equation. The paper is organized as follows: the RB-FE-HMM is introduced and discussed in Section 2. Numerical experiments confirming the theoretical convergence rates and comparison with the FE-HMM are reported in Section 3 .

\section{RB-FE-HMM for the wave equation}

The starting point of our numerical method is the FEM with numerical integration for the wave equation. We denote $\mathcal{T}_{H}$ a macro partition of $\Omega$ in simplicial or quadrilateral elements $\mathrm{K}$, where the meshsize $H:=\max _{K \in \mathcal{T}_{H}} \operatorname{diam}(K)$ is independent of $\varepsilon$ and in particular $H \gg \varepsilon$ is allowed. For an integer $\ell \geq 1$, we define the macro FE space on $\mathcal{T}_{H}$,

$$
S_{0}^{\ell}\left(\Omega, \mathcal{T}_{H}\right)=\left\{v^{H} \in H_{0}^{1}(\Omega):\left.v^{H}\right|_{K} \in \mathcal{R}^{\ell}(K) \quad \forall K \in \mathcal{T}_{H}\right\},
$$


where $\mathcal{R}^{\ell}(K)$ is chosen either $\mathcal{P}^{\ell}(K)$ the space of polynomials of degree at most $\ell$ for simplicial elements, or $\mathcal{Q}^{\ell}(K)$ the space of polynomials of degree at most $\ell$ in each variable for quadrilateral elements. For each $K \in \mathcal{T}_{H}$, we define a quadrature formula (QF) $\left\{\omega_{K j}, x_{K}\right\}_{j=1}^{J}$ and assume that the QF satisfies the standard hypotheses ensuring optimal convergence rates of the FEM in elliptic problems (see [10]). The FEM with numerical integration for the wave equation reads: find $u^{H}(t):[0, T] \rightarrow S_{0}^{\ell}\left(\Omega, \mathcal{T}_{H}\right)$, such that

$$
\left(\partial_{t}^{2} u^{H}(t), v^{H}\right)+B_{H}\left(u^{H}(t), v^{H}\right)=f_{H}\left(t ; v^{H}\right) \quad \forall v^{H} \in S_{0}^{\ell}\left(\Omega, \mathcal{T}_{H}\right),
$$

where the bilinear form $B_{H}(\cdot, \cdot)$ is defined, for $v^{H}, w^{H} \in S_{0}^{\ell}\left(\Omega, \mathcal{T}_{H}\right)$, as

$$
B_{H}\left(v^{H}, w^{H}\right)=\sum_{K \in \mathcal{T}_{H}} \sum_{1 \leq j \leq J} \omega_{K j} a^{0}\left(x_{K j}\right) \nabla v^{H}\left(x_{K j}\right) \cdot \nabla w^{H}\left(x_{K j}\right) .
$$

Here $f_{H}\left(t ; v^{H}\right)$ is an approximation of $\int_{\Omega} f(t, x) v^{H}(x) d x$ and the initial and boundary conditions are appropriate FE approximations of $g_{0}, g_{1}$.

The method above is however of no practical use in general as $a^{0}\left(x_{K j}\right)$ is unknown. In the FE-HMM presented in [1], $a^{0}\left(x_{K j}\right)$ are computed at each quadrature point by solving appropriate micro FEM on a sampling domain $K_{\delta_{j}}$ centered in $x_{K j}$. Repeated micro FEM computations can however be expensive as the macro mesh is refined. To address this issue, following [3,4], we introduce in what follows a low dimensional space spanned by appropriately chosen reduced basis to compute an approximation of each $a^{0}\left(x_{K j}\right)$. The RB algorithm is divided in an offline and online stage. While the offline stage is usually only performed once, the output of this process can be used repeatedly in different online procedures, e.g.,

- for adaptive macro mesh refinement refinements [4];

- for different macro solvers (FEMs, finite difference methods, etc.);

- for different source terms, boundary conditions, or initial conditions;

- for different model equations with the same multiscale tensor (stationary or time-dependent problems).

\subsection{RB-FE-HMM: online stage}

We first assume that a set of RB functions $\left\{\xi_{1}, \cdots, \xi_{N}\right\}$ and the corresponding RB space

$$
S_{N}(Y):=\operatorname{span}\left\{\xi_{1}, \cdots, \xi_{N}\right\},
$$

are already obtained from an offline stage (described in the next subsection) and describe the corresponding macroscopic approximation. We first recall that a fundamental condition for the efficiency of the RB-FE-HMM is that the tensor $a^{\varepsilon}$ has the following affine representation,

$$
a_{x}(y)=\sum_{1 \leq p \leq P} \Theta_{p}(x) a_{p}(y), \forall y \in Y,
$$


where $y:=\frac{x}{\varepsilon} \in Y=(-1 / 2,1 / 2)^{d}$. We note that when (2.6) is not readily available, one can use the so-called empirical interpolation method (EIM) [6] to provide an affine approximation of the tensor $a^{\varepsilon}$.

In order to compute an approximation $a_{N}^{0}(x)$ of $a^{0}\left(x_{K j}\right)$ at a given quadrature point $x_{K j}$, we consider the RB-FE-HMM online cell problems: find $\psi_{N, \tau}(y)=\sum_{j=1}^{N} \alpha_{\tau, j} \xi_{j} \in S_{N}(Y)$ for $\boldsymbol{\alpha}_{\tau}=\left(\alpha_{\tau, 1}, \cdots, \alpha_{\tau, N}\right) \in \mathbb{R}^{N}$, such that

$$
\int_{Y} a_{x}(y) \nabla \psi_{N, \tau}(y) \cdot \nabla z_{N} d y=-\int_{Y} a_{x}(y) \mathbf{e}_{m} \cdot \nabla z_{N} d y, \forall z_{N} \in S_{N}(Y) .
$$

where $\left\{\mathbf{e}_{m}\right\}_{m=1}^{d}$ is the canonical basis of $\mathbb{R}^{d}$ and $\tau$ is the parameter index $\tau=(x, m)$. We see that the cell problems are parametrized by the location $x \in \Omega$ where we want an approximation of $a^{0}(x)$ and the index $m$ of the canonical basis in $\mathbf{e}_{m} \in \mathbb{R}^{d} .{ }^{1}$ For problems with multiscale tensors of form $a^{\varepsilon}(x, t)$, the micro problems can be similarly parametrized by $(x, t)$. In this case, the $\Theta_{p}$ functions in (2.6) are of form $\Theta_{p}(x, t)$ and the parameter index in (2.7) is modified as $\tau=(x, t, m)$.

Using (2.6), we can write (2.7) as the following linear system

$$
\left(\sum_{1 \leq p \leq P} \Theta_{p}(x) A_{p}\right) \boldsymbol{\alpha}_{\tau}=-\sum_{1 \leq p \leq P} \Theta_{p}(x) F_{p, m},
$$

where

$$
\left(A_{p}\right)_{m n}=\int_{Y} a_{p}(y) \nabla \xi_{m}(y) \cdot \nabla \xi_{n}(y) d y, \quad\left(F_{p, m}\right)_{i}=\int_{Y} a_{p}(y) \mathbf{e}_{m} \cdot \nabla \xi_{i}(y) d y,
$$

are precomputed and stored in the offline stage. The unknown tensor $a^{0}(x)$ is then estimated from the micro solution $\psi_{N, \tau}$ as

$$
\begin{aligned}
\left(a_{N}^{0}(x)\right)_{m n} & =\int_{Y} a_{x}(y)\left(\nabla \psi_{N, \tau}(y)+\mathbf{e}_{m}\right) \cdot \mathbf{e}_{n} d y, \\
& =\sum_{1 \leq p \leq P} \Theta_{q}(x)\left(\boldsymbol{\alpha}_{\tau} \cdot F_{p, n}+\left(G_{p}\right)_{m n}\right), \quad m, n=1, \cdots, d,
\end{aligned}
$$

where $\left(G_{p}\right)_{m n}=\int_{Y}\left(a_{p}(y)\right)_{m n} d y$ is also precomputed in the offline stage.

Comparison with the FE-HMM. In the FE-HMM, the micro problems (2.7) are solved in a micro FE space $S^{q}\left(K_{\delta_{j}}, \mathcal{T}_{h}\right)$ coupled with periodic or Dirichlet boundary conditions. The FE space $S^{q}\left(K_{\delta_{j}}, \mathcal{T}_{h}\right)$ is defined on the cell domain $K_{\delta j}:=x_{K j}+\delta Y, \delta \geq \varepsilon, \forall K \in \mathcal{T}_{H}$ equipped with a micro mesh size $h / \varepsilon=\hat{h}$ and a FEM with piecewise polynomials of degree $q$. Hence solving a micro cell

\footnotetext{
${ }^{1}$ We recall that for classical homogenization (e.g., for periodic tensors) the homogenized tensor $a^{0}$ relies on the solution of $d$ cell problems with a right-hand side of the form $a(y) \mathbf{e}_{m}[7]$.
} 
problem in the FE-HMM is equivalent to solving a linear system with DOF $\mathcal{O}\left(\hat{h}^{-d}\right)$, where $\hat{h}$ must be refined simultaneously to the macro meshsize $H$ for optimal accuracy [2]. In contrast, we note that (2.7) only involves the solution of $N \times N$ linear system (2.8) with $N$ fixed and usually small [3,4]. As can be seen in our numerical experiments, the online solver of the RB-FE-HMM for the wave equation is much faster than the FE-HMM.

\subsection{RB-FE-HMM: offline stage}

The RB space is constructed in an offline stage, where a small number of representative micro problems which are parametrized by the macro locations and the canonical basis $\mathbf{e}_{m}$, are selected by a greedy algorithm with accuracy controlled by an a posteriori error estimator. The key steps of the offline procedure are the following.

1. Initial step: For a given offline tolerance tol and a natural number $N_{\text {train }}$ we consider a randomly chosen training set

$$
\Xi_{\text {train }}=\left\{\left(x_{n}, \mathbf{e}_{\eta}\right) ; x_{n} \in \Omega, n=1, \cdots, N_{\text {train }}, \eta=1, \cdots, d\right\} .
$$

2. Iteration step: Assuming that the basis functions $\left\{\xi_{1}, \cdots, \xi_{l-1}\right\}$ are already computed we compute the next $\mathrm{RB}$ function $\xi_{l}$ as described below.

a. Select the next target cell problem by an a posteriori estimator $\Delta_{l-1, n}^{\eta}$ :

$$
\left(x_{l}, \mathbf{e}_{l}\right)=\operatorname{argmax}_{\left(x_{n}, \mathbf{e}_{\eta}\right) \in \Xi_{\text {train }}} \Delta_{l-1, n}^{\eta} .
$$

If $\max _{\left(x_{n}, \mathbf{e}_{\eta}\right)} \Delta_{l-1, n}^{\eta} \leq$ tol then go to step 3 .

b. Compute the cell problem (2.7) located at $x_{l}$ with right-hand side based on $\mathbf{e}_{l}$ with an accurate $F E$ solver in order to have a negligible offline FE discretization error. Add the cell solution to the RB basis $\left\{\xi_{1}, \cdots, \xi_{l-1}\right\}$ after an orthogonalization process.

3. Store the offline output: $A_{p}, F_{p, m}, G_{p}$, see (2.8),(2.9).

An appropriate a posteriori estimator is crucial for the offline process and we refer to [3] for details. The accuracy of the RB-FE-HMM for the wave equation is described in the following theorem that can be proved by combining the a priori analyses in [1] and [3].

Theorem 1. Under appropriate regularity assumptions for the homogenized solution $u^{0}$, the following estimate for the RB-FE-HMM solution $u^{H}$ holds,

$$
\left\|u^{H}-u^{0}\right\|_{L^{\infty}\left(0, T ; H^{1-\mu}\right)(\Omega)} \leq C\left(H^{\ell+\mu}+e r r_{R B}+e r r_{m o d}\right), \quad \mu=0,1,
$$

where $H^{0}(\Omega):=L^{2}(\Omega)$, e $e_{\text {mod }}$ is the HMM modeling error analyzed in [1] and $\operatorname{err}_{R B} \leq C\left(\frac{1}{\mathcal{N}^{q}}+r_{R B}\right)$, where $\mathcal{N}$ is the DOF of the offline FEM used to compute the $R B$.

As analyzed in [3], provided an exponential Kolmogorov $N$-width decay for the best $N$-dimensional approximation subspace of the infinite dimensional 
space of "cell solutions", the RB a priori error $r_{R B}$ can be bounded as $r_{R B} \leq$ $C e^{-s N}$, where $s$ is a positive constant and $N=\operatorname{dim}\left(S_{N}(Y)\right)$. As can be seen in our numerical tests the offline error $\operatorname{err}_{R B}$ is often negligible compared to the macro error $\mathcal{O}\left(H^{\ell+\mu}\right)$. Finally we note that the RB error $r_{R B}$ can also be controlled by the a posteriori error estimator $[17,8,3]$.

\section{$3 \quad$ Numerical examples}

In this section, we consider the model equation (1.1) in $\Omega \times[0, T]=[0,1]^{2} \times$ $[0,0.1]$ and show two numerical examples to verify the performance of the RB-FE-HMM for wave problems in heterogeneous media. In the following, we choose $\varepsilon=10^{-3}$ and fix the micro sampling domain size $\delta=\varepsilon$. We set $f=0$ in (1.1) and choose initial condition as $g_{0}(x)=0.1 \exp \left(\frac{|x-c|^{2}}{\sigma^{2}}\right), g_{1}(x)=$ $0, \sigma=0.1, c=(0.5,0.5)$. We apply the P1 FEM for the space discretization (for both the offline and the online procedures) and the leap frog scheme for the time discretization. The implementation is done in Matlab 2012a without using a parallel implementation.

$2 D$ problem with a continuous tensor. We consider the following diagonal tensor applied to (1.1) (also used for the elliptic test problem in [3])

$$
\begin{aligned}
& a_{11}^{\varepsilon}(x)=x_{1}^{2}+0.2+\left(x_{2}+1\right)\left(\sin \left(2 \pi x_{1} / \varepsilon\right)+2\right) \\
& a_{22}^{\varepsilon}(x)=x_{2}^{2}+0.05+\left(x_{1} x_{2}+1\right)\left(\sin \left(2 \pi x_{2} / \varepsilon\right)+2\right) .
\end{aligned}
$$

As we noticed before, the same offline data can be used for different model equations. Therefore we reuse the offline output from the first example in [3, Section 5] for our wave equation. The details of the offline stage can be seen in [3, Section 5] and we just mention here that 10 reduced bases are obtained in the end of the offline stage which are solved on a $1500 \times 1500$ mesh. The offline CPU time is 1045 seconds. The fast decay of the a posteriori error in the offline stage in shown in Fig. 1.

In the online stage, we set the time step $\Delta t=0.1 H$ to insure stability of the time integrator, where $H=1 / N_{M A C}$ and $N_{M A C}$ denotes the DOF in each space direction. We test the error $\left\|u^{H}-u^{r e f}\right\|$ in $H^{1}$ and $L^{2}$ norms as well as the CPU time for both the RB-FE-HMM online stage and the FE-HMM. A reference solution $u^{r e f}$ is computed from the homogenized equation with tensor $a^{0}$ by P1 FEM on a $256 \times 256$ uniform mesh. As shown in Table 1, the RB-FE-HMM and the FE-HMM have almost the same accuracy but the CPU time comparison shows great efficiency advantage using the RB-FE-HMM.

$2 D$ problem with a discontinuous tensor. In this test, we use a discontinuous multiscale tensor as shown in Fig. 2(a) for the multiscale wave equation. This tensor is also considered for elliptic equation in [3, Section 5]. As we can see in Fig. 2(b), the $H^{1}$ and $L^{2}$ errors converge with the rate $\mathcal{O}(H)$ and $\mathcal{O}\left(H^{2}\right)$ 


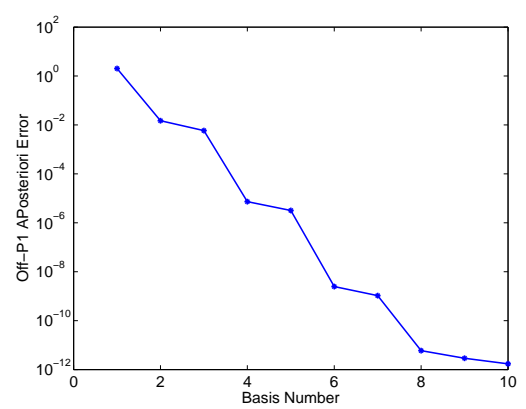

Fig.1: The decay of the a posteriori error versus reduced basis numbers [3].

Table 1: Comparison between RB-FE-HMM and FE-HMM (the micro DOF $N_{M I C}$ is set to $\left.N_{M I C}=N_{M A C}\right)$.

\begin{tabular}{c|c|c|c||c|c|c}
\hline & \multicolumn{3}{|c||}{ RB-FE-HMM } & \multicolumn{3}{c}{ FE-HMM } \\
\hline$N_{\text {M AC }}$ & $H^{1}$ Error & $L^{2}$ Error & CPU (s) & $H^{1}$ Error & $L^{2}$ Error & CPU (s) \\
\hline 16 & 0.0717 & $2.2 \mathrm{e}-3$ & 0.45 & 0.0718 & $2.2 \mathrm{e}-3$ & 2.8 \\
32 & 0.0350 & $6.5 \mathrm{e}-4$ & 0.9 & 0.0350 & $6.5 \mathrm{e}-4$ & 28 \\
64 & 0.0173 & $1.6 \mathrm{e}-4$ & 6.3 & 0.0173 & $1.6 \mathrm{e}-4$ & 512 \\
128 & 0.0077 & $3.2 \mathrm{e}-5$ & 26 & 0.0077 & $3.2 \mathrm{e}-5$ & 8691 \\
\hline
\end{tabular}

respectively. The RB error $\operatorname{err}_{R B}$ is negligible here and cannot be observed as the macro discretization error dominates. The convergence rates corroborate the a priori error estimate given in Theorem 1.

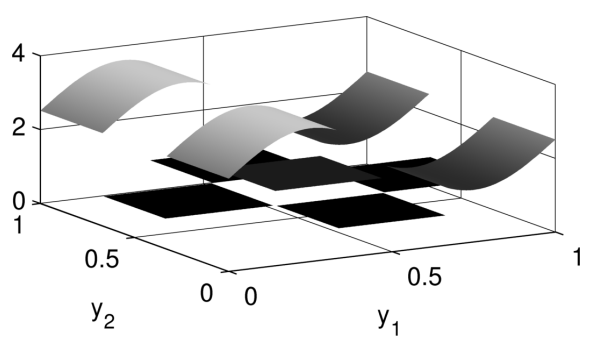

(a) $a_{11}^{\varepsilon}(\bar{x}, \cdot), \bar{x}=(0.1,0.1)$

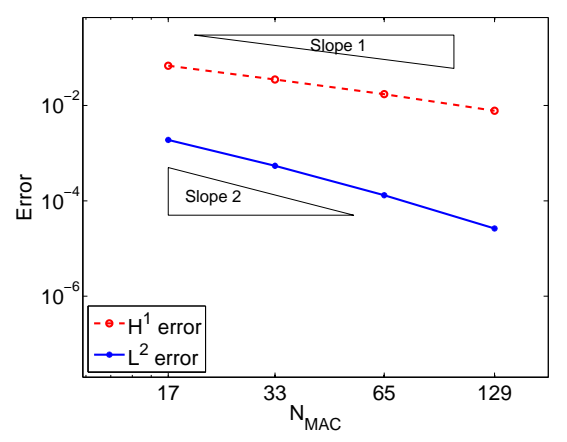

(b) $\left\|u^{H}-u^{r e f}\right\|$

Fig.2: (a) shows $a_{11}^{\varepsilon}(\bar{x}, \cdot)$, in reference sampling domain $Y$. (b) shows $H^{1}$ and $L^{2}$ errors with $u^{r e f}$ computed by the FE-HMM $\left(N_{M A C}=N_{M I C}=256\right)$. 


\section{References}

1. A. Abdulle And M. Grote, Finite element heterogeneous multiscale method for the wave equation, SIAM, Multiscale Model. Simul. 9:2 (2011), 766-792.

2. A. ABDulle, On a priori error analysis of fully discrete heterogeneous multiscale FEM, SIAM, Multiscale Model. Simul. 4:2 (2005), 447-459.

3. A. ABDulle AND Y. BAI, Reduced basis finite element heterogeneous multiscale method for high-order discretizations of elliptic homogenization problems, J. Comput. Phys. 231:21 (2012), 7014-7036.

4. _ Adaptive reduced basis finite element heterogeneous multiscale method, Comput. Methods Appl. Mech. Engrg. 257 (2013), 201-220.

5. A. Abdulle, W. E, B. Engquist, and E. Vanden-Eijnden, The heterogeneous multiscale method, Acta Numer. 21 (2012), 1-87.

6. M. Barrault, Y. Maday, N. Nguyen, and A. Patera, An 'empirical interpolation method': Application to efficient reduced-basis discretization of partial differential equations, C. R. Acad. Sci. Paris Ser.I 339 (2004), 667-672.

7. A. Bensoussan, J.-L. Lions, And G. Papanicolaou, Asymptotic analysis for periodic structures, North-Holland Publishing Co., Amsterdam, 1978.

8. S. BOYAVAL, Reduced-basis approach for homogenization beyond the periodic setting, Multiscale Model. Simul. 7:1 (2008), 466-494.

9. S. Brahim-Otsmane, G. A. Francfort, and F. Murat, Correctors for the homogenization of the wave and heat equations, J. Math. Pures Appl. 71:3 (1992), 197-231.

10. P. CiARlet, Basic error estimates for elliptic problems, Handbook of numerical analysis, vol. 2, Elsevier science publishers B.V., North-Holland, 1991.

11. W. E And B. EngQuist, The heterogeneous multiscale methods, Commun. Math. Sci. 1:1 (2003), 87-132.

12. W. E, P. Ming, AND P. ZhAng, Analysis of the heterogeneous multiscale method for elliptic homogenization problems, J. Amer. Math. Soc. 18:1 (2005), 121-156.

13. Y. EFEndiev AND T. Y. Hou, Multiscale finite element methods. theory and applications, Surveys and Tutorials in the Applied Mathematical Sciences, vol. 4, Springer, New York, 2009.

14. V. Jikov, S. Kozlov, AND O. Oleinik, Homogenization of differential operators and integral functionals, Springer-Verlag, Berlin, Heidelberg, 1994.

15. J. L. Lions AND E. Magenes, Problèmes aux limites non homogènes et applications, Travaux et recherches mathématiques, vol. 1, Dunod, Paris, 1968.

16. F. Murat And L. TARTAR, H-convergence, topics in the mathematical modeling of composite materials, Progr. Nonlinear Differential Equations Appl. 31 (1997), 21-43.

17. G. RozzA, D. Huynh, And A. T. PAterA, Reduced basis approximation and a posteriori error estimation for affinely parametrized elliptic coercive partial differential equations, Arch. Comput. Methods. Eng. 15 (2008), 229-275. 